\title{
Advanced Approximation of Channel Quality in a VLC CDM System
}

\author{
Gabor Szabo and Eszter Udvary
}

\begin{abstract}
Expanding the functionality of LED indoor lighting with visible light communication (VLC) allows an additional communication channel beside wireless radio in buildings. This service may be based on various channel access methods and modulation types. Code division multiplexing (CDM) is a suitable method to such an application, but it is complicated to measure the signal quality which is essential to compare different codes and settings, and necessary for some applications like position-dependent information services. Computing crest factor is a suitable method to estimate quality, but it may be inaccurate in some cases. This paper presents novel methods to approximate the quality of received CDM signals along with the crest factor, aiding the more accurate investigation of the VLC CDM technique.
\end{abstract}

Index Terms-visible light communication, code division multiplexing, signal quality, characterization, $O O C$, Gold codes

\section{INTRODUCTION}

$\mathrm{V}$ isible light communication (VLC) refers to free space optical transmission with light emitting diodes (LEDs), adding an alternative functionality to lighting or visible light indicator devices [1], [2], [3]. So these light sources, beside their main purposes, can invisibly embed data in their light output, which is immune to radio interferences, does not have environmental and human health risks, and is able to provide a high data rate connection. Due to its benefits this technology has recently attracted significant attention as a promising complementary technology for short range radio frequency communications [2], [4], [5], [6].

Using the indoor lighting system to positioning purposes via VLC may be a convenient and cost-efficient way to determine the location of portable devices, because the internal areas of a building are usually fully covered with light sources. A mobile node can be located with VLC on several different ways. With the angle of arrival (AoA) method [8] very good accuracy may be achieved. Its main disadvantage is that it requires an image sensor array, which is far more expensive and has a lower bandwidth than a single photodiode [7]. The signal traveling time measurement techniques, such as time of arrival (ToA) and time difference of arrival (TDoA), require not only ultra high speed circuits on the receiver side, but also synchronization between at least the

G. Szabo and E. Udvary are with the Department of Broadband Infocommunication and Electromagnetic Theory, Budapest University of Technology and Economics, Egry József utca 18, Budapest 1111, Hungary (e-mail: gabor.szabo@hvt.bme.hu, udvary@hvt.bme.hu a URL: http://hvt.bme.hu ) transmitters, increasing the installation and circuit costs [7]. A less accurate but cost-efficient localization method is based on the received signal strength (RSS), which eliminates the need of any synchronization and complex optical devices, [9], [10], [11].

As LEDs used in indoor lighting can be modulated efficiently only up to tens of $\mathrm{MHz}$ [12], inter-symbol interference (ISI) caused by multi-path propagation does not affect the operation. Moreover, one of the main drawbacks of the VLC - its relatively short range - can be converted into advantage in some location-dependent applications, as the distant illumination devices interfere less with the relevant signals.

In most of the applications the signals of different transmitters (lamps) have to be separated on the receiver side, so some kind of multiplexing methods should be used. With phosphor-converted white LEDs of illumination devices wavelength-division multiplexing (WDM) is not feasible without interfering with the lighting function. Using simple intensity modulation with direct detection (IM/DD) in an undivided space three fundamental multiplexing methods are possible. Time-division multiplexing (TDM) means that the participants do not communicate in the same time, but alternately, so the other communication parameters (e.g. frequency domain) may be the same for all transmitters. In the case of frequency-division multiplexing (FDM), different nodes transmit on different frequency bands even simultaneously, but this also makes the continuous reception of several signals more difficult. As for code-division multiplexing (CDM), the communication can be in the same time and on same frequency band, because the transmitters use different codes to identify their signals. This method enables the possibility of continuous communication with minimal bandwidth, which is suitable for also an indoor positioning system along with other broadband VLC services.

This paper presents the CDM technique in aspects of the usage in VLC systems, including the introduction of the main code types like optical orthogonal codes (OOCs) and pseudonoise codes. In the followings the problems of CDM channel quality approximation are negotiated, introducing two new quality indication methods. Finally, for an example, a novel quality indicator measure is used to compare noise sensitivity of different code sets with CDM.

\section{CODE Division MultipleXING}

Code division multiplexing is a digital multiplexing method based on binary code sets having special 
autocorrelation and cross-correlation properties. If the length of codes is $n$, every bit of data is converted on the transmitter side to $n$ chips. In a possible realization, the transmitter puts out its own code or its bitwise negative on every bit, according to the actual data bit value. The detector receives all signals at the same time (superposition), and correlates this compound signal with each code. The polarity of correlation peak values indicates the bits sent.

In synchronous CDM systems, where the transmitter nodes are synchronized, it is possible to use orthogonal code sets, e.g. orthogonal variable spreading factor (OVSF) codes. In this case of perfect orthogonality the crosstalk between CDM channels is zero, but the synchronization requirements make this mode not suitable for a simple VLC system.

The asynchronous CDM technique does not need any synchronization between the transmitters; it is based on quasiorthogonal codes, like the bipolar Gold codes and the unipolar OOCs. These codes are not orthogonal, but their circular cross-correlation peak is low, also having low circular autocorrelation peak. Due to the lack of orthogonality there is some crosstalk between the CDM channels, but using appropriate codes the signal-to-interference-plus-noise-ratio (SINR) can be high enough to achieve good results.

Our experiments are focused on asynchronous CDM transmission due to the suitability for VLC systems.

\section{OPticAl ORTHOGONAL CODES}

OOCs are unipolar quasi-orthogonal binary codes which can be represented with four parameters, usually written in the following format: (n, w, $\lambda_{\mathrm{a}}, \lambda_{\mathrm{c}}$ ), where $n$ is the code length, $w$ is the code weight (number of ones), $\lambda_{\mathrm{a}}$ is the upper bound of the circular autocorrelation function, $\lambda_{\mathrm{c}}$ is the upper bound of the circular cross-correlation function between the elements of the code set [13].

$$
\begin{gathered}
C_{x x}(\tau)=\sum_{i=0}^{n-1} x_{i} x_{i \oplus \tau}= \begin{cases}w, & \tau=0 \\
\leq \lambda_{a}, & 1 \leq \tau \leq n-1\end{cases} \\
C_{x y}(\tau)=\sum_{i=0}^{n-1} x_{i} y_{i \oplus \tau} \leq \lambda_{c} \quad 0 \leq \tau \leq n-1
\end{gathered}
$$

Unipolarity means that the codes comprise zeros (' 0 ') and ones (' 1 '), in contrast with bipolar codes, which consist of '1 ' and ' +1 ' elements. Due to this fact the minimum value of OOC correlation parameters is 1 , slightly limiting the maximal SINR compared to bipolar code sets. Nevertheless, the algorithmic efficiency of an OOC correlator can be very good especially for low weight OOCs, because a point of the correlation function is virtually just a sum of $w$ values (multiplication with ones and zeros). This is an advantage when using a low performance portable device for VLC CDM indoor localization.

It is important to mention that the inverse of an OOC (ones and zeros swapped) is not guaranteed to have as good correlation properties as the original. This is particularly right correlation properties as the original. This is particularly right for low weight OOCs: inverting a sequence of few ones and lots of zeros would obviously lead to a $\lambda_{\mathrm{a}}$ which is almost $n$. So the data transmission method using a code and its inverse as two different symbols is not feasible with OOCs.

\section{Bipolar Pseudo Noise Codes}

A bipolar binary code differs from a unipolar one in its elements: it is a sequence of ' -1 ' and ' +1 ' chips. A pseudo noise $(\mathrm{PN})$ code of this type has a spectrum similar to a random binary sequence, but it is deterministically generated. Of course, when using in a CDM system, we must introduce similar restrictions in the correlation properties of a code set as in OOCs.

A general format for PN codes is $\left(\mathrm{n}, \lambda_{\mathrm{a}}, \lambda_{\mathrm{c}}\right)$, where $n$ is the code length, $\lambda_{a}$ is the upper bound of the circular autocorrelation function, $\lambda_{\mathrm{c}}$ is the upper bound of the circular cross-correlation function between the elements of the code set. The code weight is not a relevant parameter for PN codes, because the number of $-1 \mathrm{~s}$ and $+1 \mathrm{~s}$ are nearly equal due to pseudo-random distribution.

The equations for correlation limits are similar to (1) and (2), with the exception of the maximum value of the autocorrelation function.

$$
\begin{gathered}
C_{x x}(\tau)=\sum_{i=0}^{n-1} x_{i} x_{i \oplus \tau}= \begin{cases}n, & \tau=0 \\
\leq \lambda_{a}, & 1 \leq \tau \leq n-1\end{cases} \\
C_{x y}(\tau)=\sum_{i=0}^{n-1} x_{i} y_{i \oplus \tau} \leq \lambda_{c} \quad 0 \leq \tau \leq n-1
\end{gathered}
$$

Why cannot we use low weight bipolar codes as in case of unipolar OOCs? It is not an arbitrary decision that we use PN codes when bipolar codes are needed. In a good bipolar code set bipolarity reduces the side lobes of the circular correlation functions, because positive and negative values cancel each other on summation. A low weight bipolar code faces with the same problems as an inverted low weight OOC. When almost the whole code consists of one symbol the positive-negative cancellation effect cannot work, so $\lambda_{\mathrm{a}}$ will be very close to $n$.

There are many different types of PN code sets. Maximum length sequences (sometimes also called m-sequences) can be generated with linear feedback shift registers, but additional selection of codes is required to form appropriate code sets. Other famous types such as Gold [14] and Kasami codes also based on m-sequences, but they define exact methods to generate the elements of a set from the starting codes.

It may be a question how to use bipolar codes for VLC, as the communication medium - the light intensity - can not have negative values, destructive interference can not occur between signals. This problem can be easily eliminated by DC-biasing the light source, e.g. driving the transmitter LED with a class-A amplifier. Suppose two light signals, $l_{1}$ and $l_{2}$ :

$$
\begin{aligned}
& l_{1}(t)=a_{1}+c \cdot x_{1}(t) \\
& l_{2}(t)=a_{2}+c \cdot x_{2}(t)
\end{aligned}
$$


where $a$ is the DC component, $c$ is the device-dependent linear conversion factor between the electrical signal amplitude and light intensity, $x$ is the electrical signal. Suppose that the DC bias is large enough in both signals:

$$
a \geq|c \cdot x(t)|
$$

The superposition of the light signals $\left(l_{1}+l_{2}\right)$ will be:

$$
l_{s}(t)=a_{1}+a_{2}+c \cdot\left(x_{1}(t)+x_{2}(t)\right)
$$

So this way either constructive or destructive interferences may occur while the light intensity is always non-negative.

\section{QUALITY OF AN ASYNCHRONOUS CDM SIGNAL}

Channel quality is such an important thing characterizing CDM signals as received signal strength (RSS) of TDM or FDM signals. This parameter is not only useful when comparing experimental settings and different codes, but inevitable when using a VLC CDM system also to simple indoor localization purposes.

Using either TDM or FDM if it is supposed that the noise and interference levels are steady, the RSS indicates also the quality of the signal. Being acquainted with the noise level, interference level and the RSS, signal-to-interference-plusnoise-ratio (SINR) can be computed.

The situation is more complicated in case of CDM, where multiple communication channels share the same frequency band at the same time. The existence of some signal just means that at least one CDM channel is received, and the RSS value is the total (sum) strength of all received signals. Information about a specified channel may be acquired only from the corresponding pre-decoded data stream. This term is introduced (and used also in the followings) for the output data of the moving window correlation between the input compound CDM signal and a channel code:

$$
\mathrm{PDDS}_{i}=\sum_{j=1}^{\text {clen }} \mathrm{CCSS}_{i+j-1} \times C O D E_{j}
$$

where $P D D S$ is the pre-decoded data stream of the channel, $C C S S$ is the compound CDM signal stream, CODE is the channel code and clen is the channel code length.

There is an example for a pre-decoded data stream of an asynchronous CDM communication on Fig. 1. Due to the lack of synchronization the starting position ("s") of the right correlation peaks (" $r$ ") is random, and $4 \mathrm{x}$ oversampling is used to reduce the chip sampling uncertainty. So with the code length of 511 one bit consists of $511 \times 4=2044$ samples (" $a$ "). The presence of positive and negative right correlation peaks indicates bipolar encoding mode, this allows supposing that bipolar codes are used.

The noise (" $n$ ") around zero is originated from the interference caused by the other channels on decoding (quasiorthogonal codes). False correlation peaks (" $f$ ") may grow out of the noise, and they can mislead the bit restoring algorithm if they are comparable to the right peaks. So the larger the right correlation peaks compared to noise and false peaks, the better the quality of a CDM channel is.

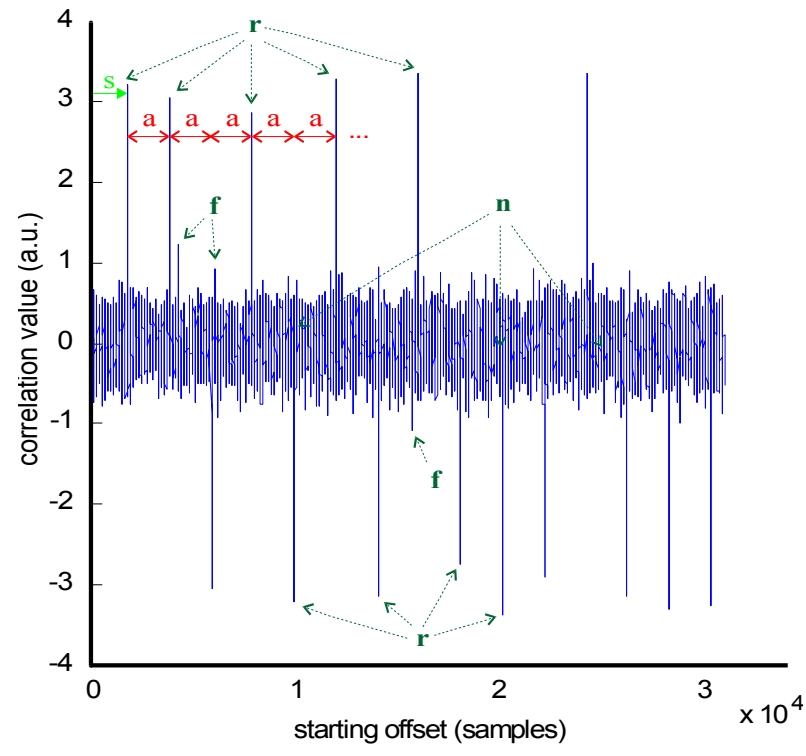

Fig. 1. Pre-decoded data stream example (bipolar PN code, length: 511, oversampling: 4x)

The next sub-sections show different methods to evaluate the quality of an asynchronous CDM signal from the predecoded data stream. It should be mentioned that any d. c. offset of the pre-decoded data should be eliminated regardless of the chosen method, because it contains no information, but it may also corrupt the calculation. This is considered done in the followings.

\section{A. Crest Factor}

Computing the crest factor of the pre-decoded data stream is a simple method to estimate the signal quality of a CDM channel. The formula for a finite-sized block of the data stream is

$$
C=\frac{|x|_{\text {peak }}}{\sqrt{\frac{1}{n} \sum_{i=1}^{n} x_{i}^{2}}}
$$

where $|x|_{\text {peak }}$ is the absolute maximum value in the block, $x_{i}$ is the $i$-th element of the block and $n$ is the block size (element count).

This measure often gives a good estimation for the signal quality, but it has a problem in some special circumstances. It may also indicate good signal quality when the stream is full of false peaks what jeopardizes the success of decoding the bits. However, computing the crest factor does not require preliminary knowledge about the CDM signal, and the resource requirements of the calculation are fairly low.

Fig. 2 and Fig. 3 are an example for a case where the crest factor does not indicate the signal quality properly - as recently mentioned. Both pre-decoded data stream have a crest factor of $\approx 9.2$ despite the visible difference in quality: the signal represented by Fig. 2 was significantly better. 


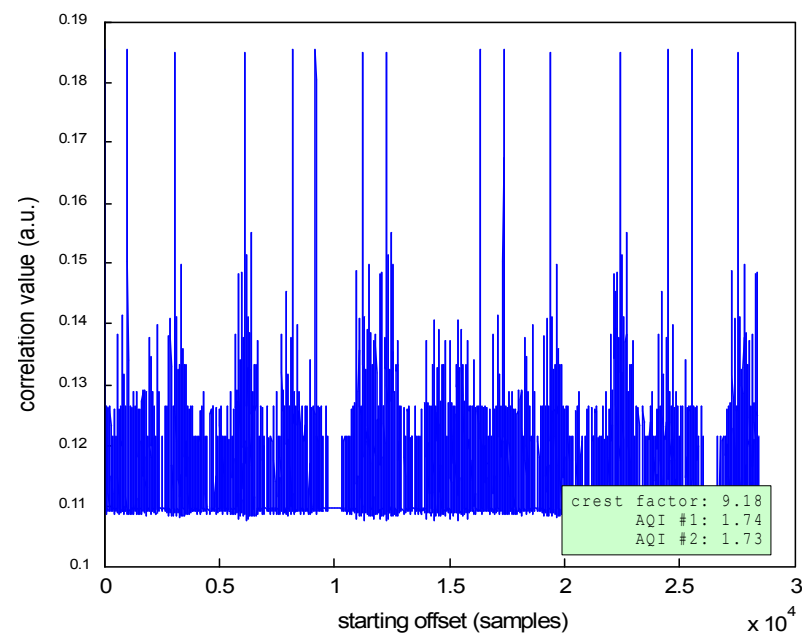

Fig. 2. Pre-decoded data stream example with computed quality indicator values (unipolar OOC, length: 255, oversampling: $4 \mathrm{x}$ )

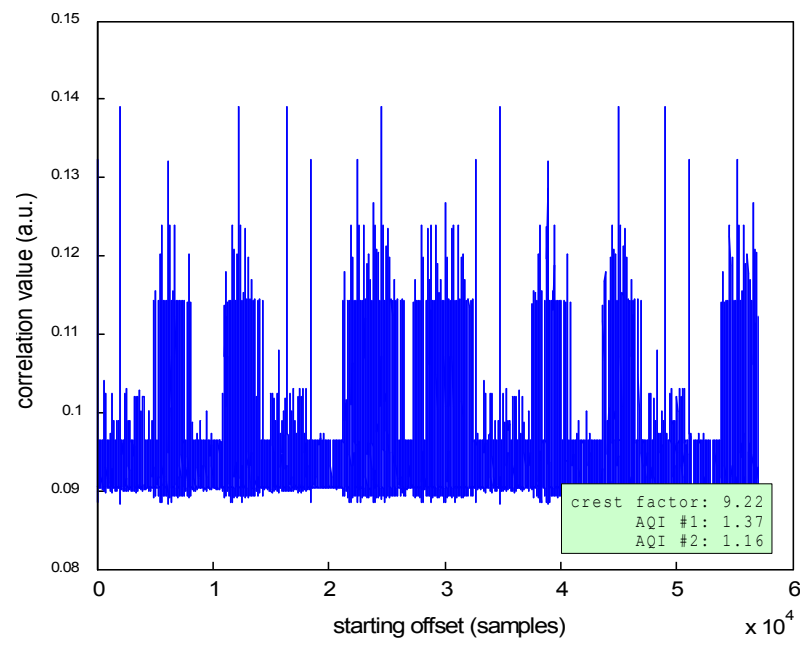

Fig. 3. Pre-decoded data stream example with computed quality indicator values (unipolar OOC, length: 511, oversampling: $4 \mathrm{x}$ )

\section{B. Advanced Quality Indicator \#1}

Knowing the parameters of the CDM signal allows more sophisticated computation to estimate the signal quality of a CDM channel. We developed two new measures to eliminate the false peak problem of crest factor. The less strict quantity called Advanced Quality Indicator \#1 (AQI1) can be calculated with the following steps.

1. Suppose that the absolute maximum value $m$ in the predecoded data stream is a (right) correlation peak.

2. When the sample rate of the transmitter and the receiver are equal, the correlation peak interval is $O V S F \times n$, where $O V S F$ is the oversampling factor (samples per chip) and $n$ is the code length. All possible locations of the correlation peaks can be computed by this peak interval and the position of the highest peak.

3. Find the highest absolute value peak $f$ that is not in the epsilon neighborhood of any previously computed correlation peak locations. Consider $f$ the highest false correlation peak. (Epsilon radius allows a minor sample rate deviation between the transmitter and the receiver.)

4. AQI1 is the absolute value ratio of the highest right correlation peak value and this highest false peak value $(A Q I 1=|m| /|f|)$.

So AQI1 indicates how close are the false correlation peaks to be mis-identified as the highest right peak. Using the presented computation method the worst AQI1 value is 1 .

In contrast to the crest factor, AQI1 is significantly different in case of the previous examples on Fig. 2 and Fig. 3 . The AQI1 of the better quality CDM channel is 1.74 , which is $\approx 27 \%$ higher than the other value.

\section{Advanced Quality Indicator \#2}

The Advanced Quality Indicator \#2 (AQI2) is a variant of AQI1, which compares the highest false correlation peak to the lowest right peak that is bigger than it. So AQI2 will be large only if most right correlation peaks are much bigger than the highest false peak. Though, a good AQI2 value neither guarantees that some right peaks are lower than the highest false peak. The calculation steps of AQI2 are below.

1. Suppose that the absolute maximum value $m$ in the predecoded data stream is a (right) correlation peak.

2. When the sample rate of the transmitter and the receiver are equal, the correlation peak interval is $O V S F \times n$, where $O V S F$ is the oversampling factor (samples per chip) and $n$ is the code length. All possible locations of the correlation peaks can be computed by this peak interval and the position of the highest peak.

3. Find the highest absolute value peak $f$ that is not in the epsilon neighborhood of any previously computed correlation peak locations. Consider $f$ the highest false correlation peak. (Epsilon radius allows a minor sample rate deviation between the transmitter and the receiver.)

4. Find the highest absolute value peaks locally in the epsilon neighborhood of all previously computed correlation peak locations. Select the lowest absolute value $z$ that is greater than or equal to $f$.

5. AQI2 is the absolute value ratio of this right correlation peak value and the highest false peak value $(A Q I 2=|z| /|f|)$.

As it is in the examples on Fig. 2 and Fig. 3, AQI2 shows the difference between the two channels even better than AQI1. The AQI2 of the better quality CDM channel is 1.73 , which is $\approx 49 \%$ higher than the other value.

\section{COMPARISON OF CODES With SIMUlation Using AQIS}

A VLC CDM system can be implemented with several types of code sets as it is discussed formerly, but these code sets may not perform similarly in all circumstances. The introduced new measures allow a more reliable comparison between the performances of different codes. In case of a general comparison AQI1 is recommended. AQI2 is a stricter indicator, so it makes less difference between closely similar lower quality channels.

In this section two different code set types are compared with AQI1 in aspect of their noise immunity: low weight 
OOCs and Gold codes. The results of multiple actual code sets of both types are averaged on the diagrams, which are presented in function of the code length. Although the codes were simulated at several noise levels, the representation would have too many dimensions plotting the full scale (AQI1 in function of code type, code length and noise level), so the results are presented in separate graphs of selected noise levels.

Before looking at the results, it is important to talk about CDM "unipolar mode" and "bipolar mode". In case of unipolar mode for one symbol (e.g. ' 1 ') the code will be transmitted, for the other (e.g. ' 0 ') only zeros will be output. At receiver side the signal is treated as unsigned. But in bipolar mode for one symbol the code itself, for the other the negative of the code will be transmitted. At receiver side the signal is treated as signed. Bipolar codes may give good results in both modes, but unbalanced (low weight) unipolar codes like OOCs perform well only in unipolar mode. This is the reason why bipolar codes are examined in unipolar and bipolar mode and OOCs only in unipolar mode.

To discover the typical noise immunity of code set types, a zero noise simulation should be done first. The further results then may be given in relative values to demonstrate the actual deterioration of signals caused by the noise. Examples for the interpretation of relative values (AQI1 ratios): 1.0 means that the current AQI1 value is equal to the noise-free AQI1 value, 0.6 means that the current AQI1 value is 60 percent of the noise-free AQI1 value etc. So a higher value shows better noise immunity.

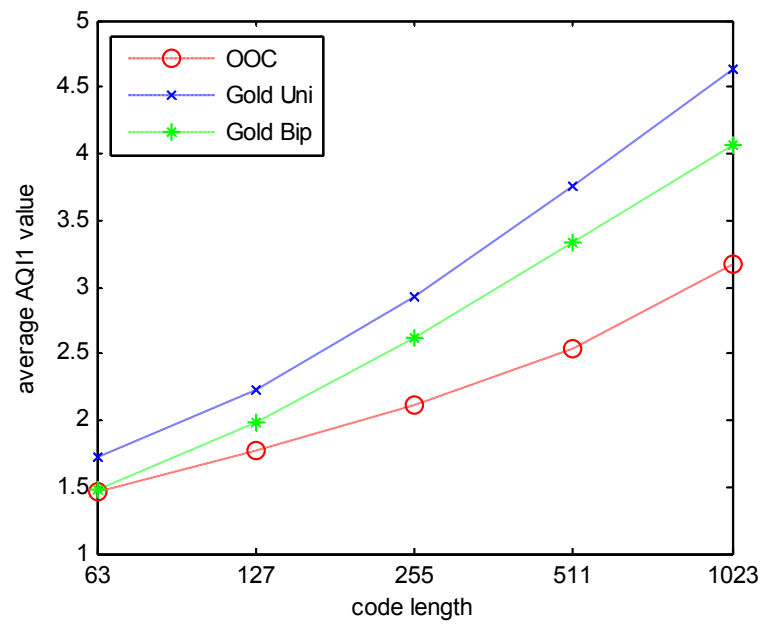

Fig. 4. The average AQI1 values with code sets of various lengths, noise-free simulation

Fig. 4 shows the average AQI1 values in case of zero noise. The Gold codes are proved to perform better, especially at longer code lengths. It may be a question why are Gold codes in unipolar CDM mode better than in bipolar mode, despite the fact that Gold codes are bipolar codes. It is a possible answer that CDM unipolar mode encodes one of two symbols with zeros (no actual transmission), so the other symbols represented by the code itself often have "quiet neighbors" that reduces the false peak levels on correlation. So the result will be higher channel quality.

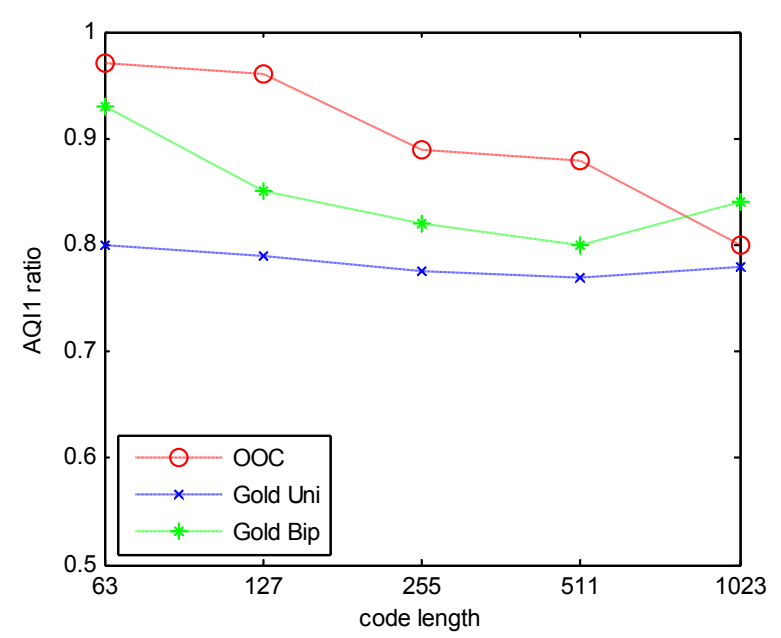

Fig. 5. The noisy vs. noise-free AQI1 ratio with code sets of various lengths, $\mathrm{SNR}=6 \mathrm{~dB}$ simulation

However as the AQI1 ratio can be seen on Fig. 5 and Fig. 6 , the ranking is quite different compared to the previous graph. Increasing the code length the achievable best correlation properties of OOCs improve at a lower rate than Gold codes (can be observed also on Fig. 4). But the deteriorative effect of the noise is also increases with the code length. In case of OOCs the negative effect may dominate, so it shows a decreasing tendency in noise immunity in function of code length despite its superiority at shorter codes, and probably underperforms the others at even longer codes (not in the graphs).

The improvement of correlation properties of Gold codes might be non-linear in function of code length, resulting in a degradation in noise immunity under 511 length, and an increment at 1023. The noise tolerance in bipolar mode proved to be better, in spite of the superiority of unipolar mode in noise-free simulation.

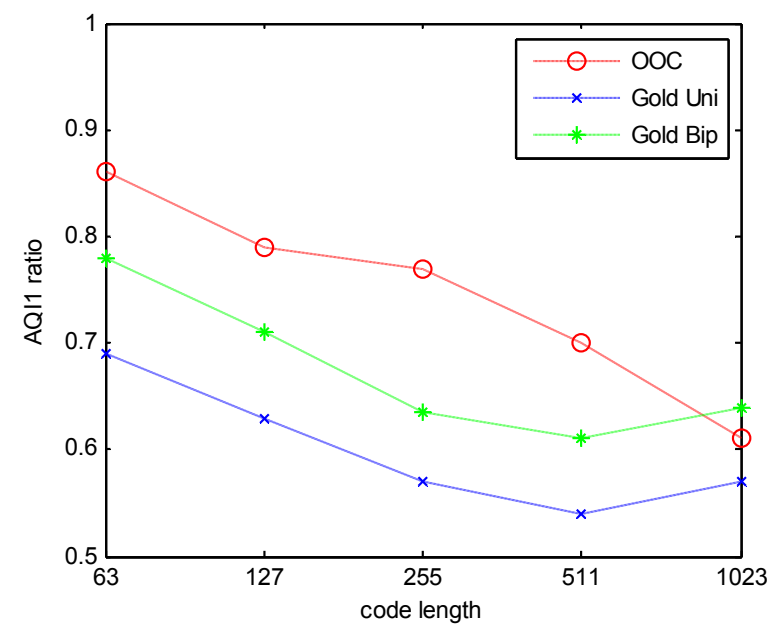

Fig. 6. The noisy vs. noise-free AQI1 ratio with code sets of various lengths, $\mathrm{SNR}=0 \mathrm{~dB}$ simulation

So the conclusion for a real noisy environment is that the longer the codes the better the Gold code sets are, but under a certain code length the performance of Gold codes and OOCs may be similar. 


\section{CONCLUSION}

In this paper the VLC related CDM problems are discussed, focusing on the asynchronous mode CDM which is more suitable for a simple VLC system. The description of the most common code set types, the unipolar OOCs and bipolar PN codes, showed the benefits, disadvantages and possibilities of these codes. Obtaining information about a CDM channel quality is not as easy as measuring RSS on a single RF signal. It was showed that the most common quality indicator measure, the crest factor, may be misleading in some cases. A possible solution is proposed for this problem, introducing two novel advanced quality indicator (AQI) measures. Computing these new measures along with the crest factor gives a better approximation to the CDM channel quality. With AQI1 the noise immunity of a CDM transmission using Gold codes and OOCs are compared, in function of the code length. These AQIs, for example, may improve the precision of a channel quality based VLC CDM indoor positioning system, and allows more reliable practical comparison between various code sets.

\section{ACKNOWLEDGMENT}

The authors would like to thank Dr. Kari Kärkkäinen from the University of Oulu, Finland for the freely downloadable bipolar pseudo-noise code bank, which was a great help for the CDM simulations.

\section{REFERENCES}

[1] B. M. Masini, A. Bazzi and A. Zanella, "Vehicular Visible Light Networks with Full Duplex Communications," IEEE International Conference on Models and Technologies for Intelligent Transportation Systems (MT-ITS), pp. 98-103, 2017.

[2] S. Randel, F. Breyer, S. C. Lee, and J. W. Walewski, "Advanced Modulation Schemes for Short-Range Optical Communications", IEEE Journal of Selected Topics in Quantum Electronics, vol. 16, no. 5, pp. 1280-1289, 2010

[3] A. Street, P. Stavrinou, D. O'brien, and D. Edwards, "Indoor optical wireless systems - a review", Optical and Quantum Electronics, vol. 29, no. 3, pp. 349-378, 1997.

[4] T. Komine and M. Nakagawa, "Integrated system of white LED visible-light communication and power-line communication", IEEE Transactions on Consumer Electronics, vol. 49, no. 1, pp. 71-79, 2003.

[5] S. Rajagopal, R. D. Roberts, and S.-K. Lim, "IEEE 802.15.7 visible light communication: modulation schemes and dimming support", IEEE Communications Magazine, vol. 50, no. 3, pp. 72-82, 2012.

[6] M. Kavehrad, "Broadband Room Service by Light", Scientific American, vol. 297, no. 1, pp. 82-87, 2007.
[7] T. Do, J. Hwang, and M. Yoo, "TDoA Based Indoor Visible Light Positioning System", Fifth International Conference on Ubiquitous and Future Networks (ICUFN), 2013.

[8] W. D. Zhong, C. Chen, H. Yang and P. Du, "Performance Analysis of Angle Diversity Multi-Element Receiver in Indoor Multi-Cell Visible Light Communication Systems", International Conference on Transparent Optical Networks (ICTON), 2017.

[9] S. Shawky, M.A. El-Shimy, Z. A. El-Sahn, M. R. M. Rizk and M. H. Aly, "Improved VLC-based Indoor Positioning System Using a Regression Approach with Conventional RSS Techniques", International Wireless Communications and Mobile Computing Conference (IWCMC), pp. 904-909, June 2017.

[10] S. Yang, E. Jeong, D. Kim, H. Kim, Y. Son, and S. Han, "Indoor three-dimensional location estimation based on LED visible light communication", Electronics Letters, vol. 49, no. 1, pp. 54-56, January 2013.

[11] S. Jung, C. Choi, S. Heo, S. Lee, and C. Park, "Received Signal Strength Ratio Based Optical Wireless Indoor Localization Using Light Emitting Diodes for Illumination", IEEE International Conference on Consumer Electronics (ICCE), pp. 63-64, January 2013.

[12] M. Mukherjee, "Wireless Communication - Moving from RF to Optical," International Conference on Computing for Sustainable Global Development (INDIACom), pp. 788-795, 2016.

[13] S. De Lausnay, L. De Strycker, J-P. Goemaere, N. Stevens, B. Nauwelaers, "Optical CDMA Codes for an Indoor Localization System using VLC", 3rd International Workshop on Optical Wireless Communications (IWOW), pp. 50-54, September 2014.

[14] R. Gold, "Optimal Binary Sequences for Spread Spectrum Multiplexing", IEEE Transactions on Information Theory, vol. 13, no. 4, pp. 619-621, October 1967.

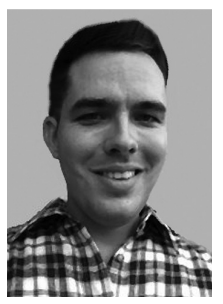

Gábor Szabó was born in Győr, Hungary, 1990 $\mathrm{He}$ received his $\mathrm{M}$. Sc. degree from the Budapest University of Technology and Economics in 2015. His research interests include optoelectronics and visible light communication.

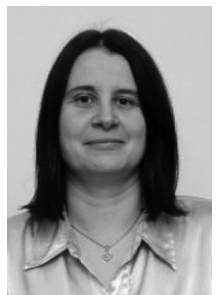

Eszter Udvary was born in Budapest, Hungary. She received her $\mathrm{Ph}$. D. degree in 2009 from Budapest University of Technology and Economics. Her research interests include microwave circuits, fiber optics and optoelectronics. 